\title{
Acute Megakaryoblastic Leukemia with Myelodysplasia- related Changes Associated with ATM Gene Deletion
}

\author{
Hiroshi Ureshino ${ }^{1,2}$, Momoka Tanabe ${ }^{2}$, Kazuya Kurogi ${ }^{2}$, \\ Masaharu Miyahara $^{2}$ and Shinya Kimura ${ }^{1}$
}

\begin{abstract}
Ataxia telangiectasia mutated (ATM) is a tumor suppressor gene, and its somatic inactivation plays a role in the pathogenesis of lymphoid malignancies. However, the role of ATM in patients with myeloid malignancies is still unknown. We herein report a case of acute megakaryoblastic leukemia (AMKL) with ATM gene deletion. An 84-year-old Japanese woman presenting with a pale face and pancytopenia was admitted to our institution and diagnosed to have AMKL with ATM gene deletion. She was treated with intravenous azacitidine. The azacitidine treatment was effective for approximately 1 year. Somatic inactivation of the ATM gene may therefore be involved in the pathogenesis of AMKL.
\end{abstract}

Key words: acute megakaryoblastic leukemia, myelodysplasia, azacitidine, ataxia telangiectasia mutated (ATM) gene

(Intern Med 55: 1625-1629, 2016)

(DOI: 10.2169/internalmedicine.55.5890)

\section{Introduction}

Acute megakaryoblastic leukemia (AMKL) is a rare subtype of acute myeloid leukemia that occurs in approximately $1.0 \%$ of all adult acute myeloid leukemia (AML) patients (1-3). AMKL is considered to be refractory AML because of both its poor response to conventional induction therapy and poor prognosis $(3,4)$. No standard treatment regimens for AMKL have so far been established.

Elderly patients with AML have poor prognoses because of such factors as the presence of comorbidities, a poor performance status, an inferior response to treatment, and generally more severe toxicities than seen in younger patients (5). Therefore, intensive chemotherapy is not recommended for elderly patients with AML, and a standard chemotherapy regimen for elderly AMKL patients thus remains to be established.

Azacitidine, a DNA methyltransferase inhibitor, has been approved for the treatment of patients with high-risk myelodysplastic syndromes (MDS). These patients include AML with $20-30 \%$ bone marrow (BM) blasts according to the
World Health Organization (WHO) criteria. Azacitidine significantly prolongs the overall survival (OS) compared with intensive chemotherapy in patients with high-risk MDS (6). Recently, Azacitidine has been used for the treatment of elderly patients with more than $30 \%$ blasts (7-10).

Ataxia telangiectasia mutated (ATM) is a tumor suppressor gene. The somatic inactivation of the ATM gene is associated with the pathogenesis of lymphoid malignancies, such as B-cell chronic lymphocytic leukemia, T-cell prolymphocytic leukemia, acute lymphoblastic leukemia, and nonHodgkin lymphoma (11). ATM gene deletion predicts the prognosis of patients with lymphoid malignancies $(12,13)$; however, the role of ATM deletion in patients with myeloid malignancies is still unknown.

We herein report a case of AMKL with ATM deletion that was successfully treated with azacitidine.

\section{Case Report}

An 84-year-old Japanese woman who presented with a pale face and general fatigue was admitted to our hospital in March 2014. Her past medical history included hyperten-

${ }^{1}$ Division of Hematology, Respiratory Medicine and Oncology, Department of Internal Medicine, Faculty of Medicine, Saga University, Japan and ${ }^{2}$ Department of Internal Medicine, Karatsu Red Cross Hospital, Japan

Received for publication June 1, 2015; Accepted for publication September 15, 2015

Correspondence to Dr. Hiroshi Ureshino, m00010hu@jichi.ac.jp 


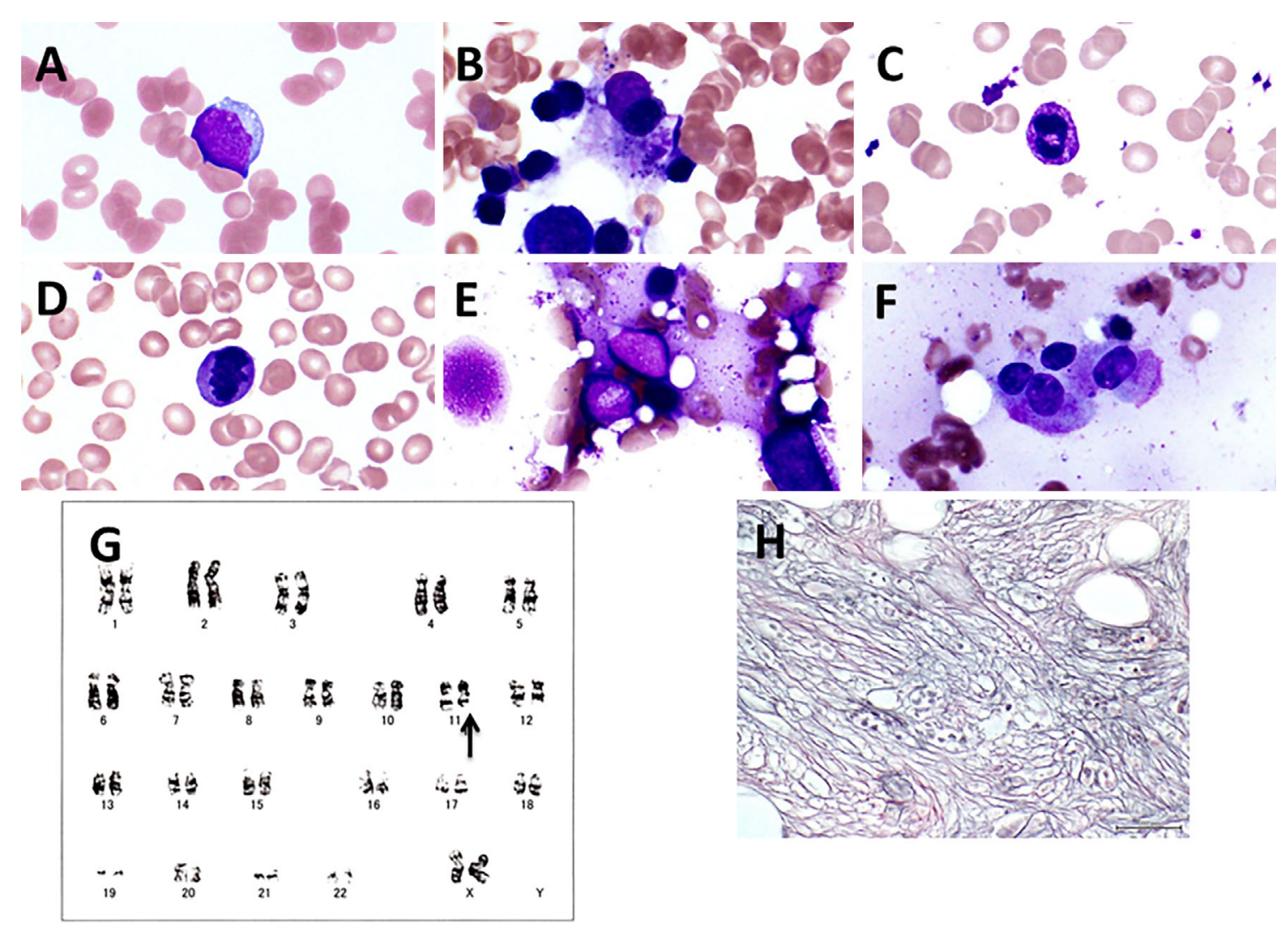

Figure 1. Bone marrow (BM) smears. A: Megakaryoblasts in the peripheral blood. B-D: The BM smear shows multi-lineage dysplasia. B: Micromegakaryocyte in the BM. C: Ring-shaped nucleus neutrophil in the BM. D: Nuclear fragmentation in erythroid cell in the BM. E, F: Megakaryoblasts and megakaryocytes in the BM. (A-F: May-Giemsa staining; original magnification $\times 1,000)$. G: Gbanded karyotype with deletion of 11q at diagnosis. $\mathrm{H}$ : Severe fibrosis in the bone marrow core (silver impregnation staining; original magnification $\times 400$ ).

sion, chronic heart failure, and cerebral infarction. According to her past history, she had not been suffered from either cytoepnia or hepatosplenomegaly. On examination, there were no obvious abnormalities, although the patient had lost vigor, and her Eastern Cooperative Oncology Group (ECOG) performance status (PS) was three.

A complete blood count and peripheral smear showed pancytopenia (hemoglobin, $47 \mathrm{~g} / \mathrm{L}$; white blood cells, 3.5× $10^{9} / \mathrm{L}$; platelets, $39 \times 10^{9} / \mathrm{L}$ ) with $29 \%$ blasts. A BM examination revealed mild hypocellularity (approximately 50\%) and severe myelofibrosis with predominantly multi-lineage dysplastic cells and leukemic blasts (The results of bone marrow smears are shown in Fig. 1).

The blasts had a high nucleus-to-cytoplasm ratio with pseudopod formation and were negative for peroxidase stain. A flow cytometric analysis to determine the immunophenotypes showed the blasts to be positive for CD13, CD34, HLA-DR, CD117, CD41, and CD61 expression, and negative for CD2, CD7, CD10, and CD19 expression. A Gbanded metaphase analysis was performed on a specimen of BM aspirate using standard cytogenetic techniques. The karyotype was 46,XX,del(11)(q) in 19/20 cells and 46,idem,i $(\mathrm{X})(\mathrm{p} 10)$ in $1 / 20$ cell (Fig. 1G). Fluorescence in situ hybridization (FISH) was performed to detect ATM deletion using a commercial probe that recognizes a $<732 \mathrm{~kb}$ region encompassing both ATM and flanking genes, which was cohybridized with a chromosome 11-specific centromere probe (Vysis LSI ATM spectrum orange and CEP 11 Alpha spectrum green, Abbott Laboratories, UK) (Fig. 2A). ATM gene deletion was recorded in $87(87 \%)$ of 100 cells scored (Fig. 2B). In contrast, mixed lineage leukemia (MLL) gene rearrangements was recorded in 0 of 100 cells $(0 \%)$ scored (Fig. 2C).

Based on the results of morphology, immunology, and cytogenetics analyses, and also according to the clinical features, the patient was diagnosed to have AMKL with myelodysplasia-related changes and ATM gene deletion.

The patient was treated with intravenous azacitidine at 75 $\mathrm{mg} / \mathrm{m}^{2}$ on days 1-7 (5) on a 28 day schedule because she could not tolerate intensive chemotherapy. After one course of azacitidine treatment, the platelet count increased to $105 x$ $10^{9} / \mathrm{L}$. The hematological improvement was not accompanied by any serious adverse events, and azacitidine administration was therefore continued. The blasts decreased gradually and were undetectable in the peripheral blood after seven cycles of treatment, although the patient required red blood cell transfusion during chemotherapy.

She had developed fever on day six after six cycles of 


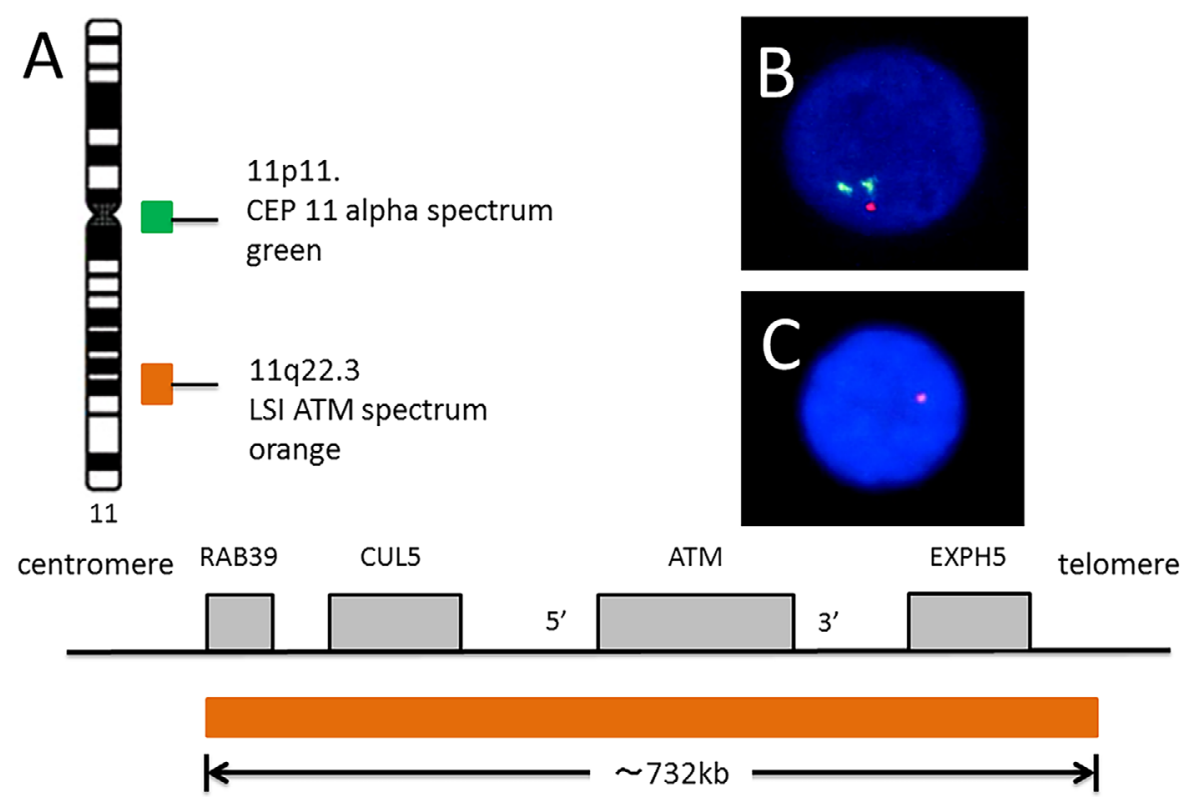

Figure 2. A: Structure of the probe designed to recognize a $<732 \mathrm{~kb}$ region encompassing both ATM and flanking genes, which was co-hybridized with a chromosome 11-specific centromere probe. B: A fluorescence in situ hybridization analysis revealed two green signals and one orange signal in the nucleous, which indicated ATM gene deletion. C: A fluorescence in situ hybridization analysis. There was only one signal and no split signal of mixed lineage leukemia (MLL) gene rearrangement, suggesting that MLL gene rearrangements was not observed.

azacitidine treatment. Cefepime was initiated, however, her fever persisted. Subsequently, we started granulocyte-colony stimulating factor (G-CSF) on day eight because she was suffering from grade 4 neutropenia. She responded favorably and her white blood cells gradually increased to $5.0 \times 10^{9} / \mathrm{L}$ on day 18 , meanwhile the blasts did not increase in number. Because leukopenia occurred after G-CSF withdrawal, we administered G-CSF continuously as necessary (Fig. 3).

A BM examination revealed a marked decrease in the blasts (from $30 \%$ to $3.1 \%$ ) in addition to myelofibrosis and several megakaryocytes after nine cycles of treatment. At that time, the peripheral blood count showed leukocytes at $3.4 \times 10^{9} / \mathrm{L}$, a hemoglobin level of $75 \mathrm{~g} / \mathrm{L}$, and a platelet count of $109 \times 10^{9} / \mathrm{L}$. Since there was no disease progression and the patient achieved a partial hematological improvement, azacitidine treatment was considered to have been effective and thus was continued (13 cycles of treatment had been completed at the time of writing this study). Meanwhile, ATM gene deletion remained in 93 (93\%) of 100 cells based on an analysis after 12 cycles of treatment. These findings suggest that a cytogenetic response had therefore not been achieved by azacitidine treatment.

\section{Discussion}

AMKL occurs frequently in children with Down syndrome (DS), in particular in those with GATA1 mutations. Meanwhile, GATA1 mutations are rare in non-DS-AMKL patients. In adults with non-DS-AMKL, deletions of chromosome $5 \mathrm{q}$ and/or chromosome $7 \mathrm{q}$ are the most frequent cytogenetic abnormalities. These cytogenetic abnormalities are associated with MDS. Approximately $90 \%$ of all AMKL patients have several cytogenetic and morphologic features that support the occurrence of AMKL as a secondary leukemia (14).

The deletion of chromosome 11q is a characteristic aberration in patients with MDS and in those with AML with myelodysplasia-related changes. MDS patients with del (11q) have a very good prognosis according to the revised international prognostic scoring system (IPSS-R) (15). The IPSS-R also predicts the outcome of patients with secondary AML evolving from MDS (16). MDS patients with del (11q) have been reported to show a smoldering clinical course and severe anemia requiring RBC transfusions (17).

Chromosome 11 aberrations and unbalanced changes such as del (11q) occur with a low frequency in AML patients. Most chromosome 11 aberrations are related to non-ATM genes, such as the MLL gene (18). There are few reports of AML in ataxia telangiectasia patients, and the somatic inactivation of the ATM gene in AML patients has not yet been reported to date (19-21). In the present case, the deletion of the ATM gene was detected by FISH and the patient had no clinical features of ataxia telangiectasia, thus suggesting that the ATM gene deletion in this case was a somatic aberration.

ATM was analyzed with a widely-used commercial probe that recognizes a $<732 \mathrm{~kb}$ region encompassing both ATM and flanking genes, which was co-hybridized with a chromosome 11-specific centromere probe designed for the screening of CLL patients (22-24). This probe is considered 


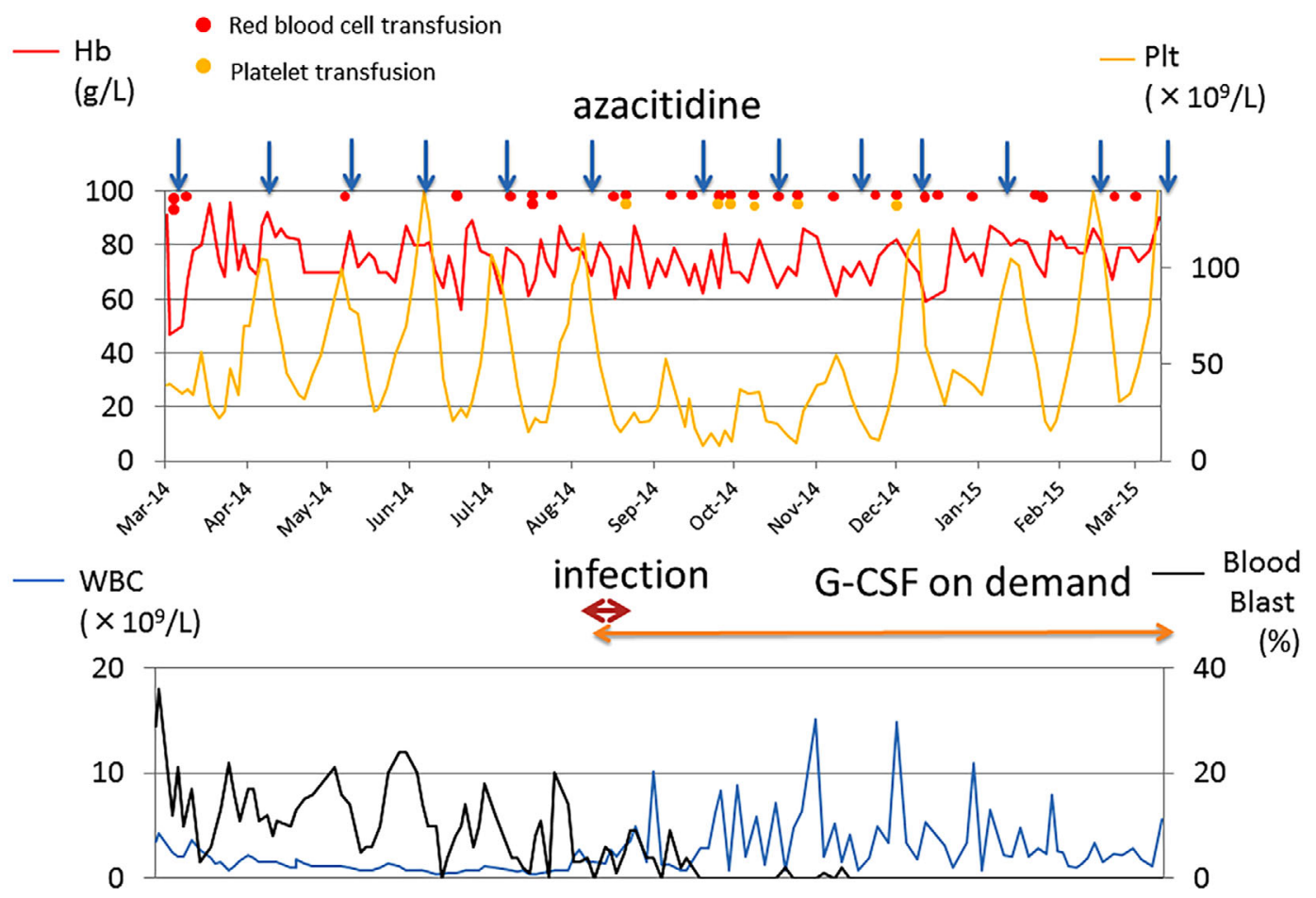

Figure 3. Clinical course: The patient was treated with intravenous azacitidine. The blasts in the peripheral blood decreased gradually and disappeared after seven cycles of azacitidine administration. There was only one episode of infection after six cycles of azacitidine treatment and no serious adverse events occurred during treatment. The patient received G-CSF on demand after seven cycles of azacitidine administration. WBC: white blood cell count, Hb: hemoglobin level, Plt: platelet count, Blood blasts: Percentage of myeloblasts in peripheral blood, G-CSF: granulocyte-colony stimulating factor

to be effective for the detection of ATM gene deletion.

The ATM gene is located on chromosome 11q22.3 to 23.1. Although there are few MDS patients with del (11q), the 11q22 band, which includes the ATM gene, is deleted in $70 \%$ of MDS patients with del (11q) (17). The present patient had ATM gene deletion and her clinical course was similar to that of MDS patients with del (11q). The effects of del (11q) may therefore be associated with the deletion of part of the ATM gene. Therefore, the clinical course of patients with del (11q) may be dependent on the inactivation of the ATM gene.

The ATM gene is a cell cycle checkpoint protein activated in response to DNA damage, and it functions as a tumor suppressor gene. The somatic inactivation of the ATM gene plays a role in the pathogenesis of lymphoid malignancies (11) and it may also be involved in the pathogenesis of myeloid malignancies.

Patients with AMKL show a poor response to conventional induction therapy and therefore tend to demonstrate a poor prognosis $(3,4)$. The prognosis of elderly patients with AML is poor because of the presence of comorbidities, a poor PS, an inferior response to treatment and more severe toxicities than seen in young patients (5). There is currently no standard treatment regimen for these patients. Azacitidine is a hypomethylating agent that has been shown to improve the prognosis of high-risk MDS and AML patients. Azaci- tidine induces DNA damage by affecting the metabolism of pyrimidine, which is involved in the activation of the ATM protein (25). The activation of the ATM protein is associated with resistance to azacitidine treatment, and the inhibition of the ATM protein induces apoptosis in azacitidine-resistant cells (26). Hence, patients with AMKL, which show somatic inactivation of the ATM gene, may be sensitive to azacitidine treatment. Azacitidine has been suggested to be a reasonable therapeutic option for most unfit AML patients $(10,27)$. The present patient was elderly and therefore intensive chemotherapy was not indicated. Azacitidine was thus considered to be a reasonable alternative treatment in the present case.

In conclusion, we herein reported the first known case of AMKL with ATM gene deletion that was successfully treated with azacitidine. The present results indicate that the somatic inactivation of the ATM gene may play a role in the pathogenesis of myeloid malignancies in addition to its involvement in lymphoid malignancies. AMKL patients with ATM gene deletion may therefore have a smoldering clinical course, favorable outcomes, and also show a good response to azacitidine treatment. Large clinical trials are necessary to confirm the present findings.

The authors state that they have no Conflict of Interest (COI). 


\section{References}

1. Tallman MS, Neuberg D, Bennett JM, et al. Acute megakaryocytic leukemia: the Eastern Cooperative Oncology Group experience. Blood 96: 2405-2411, 2000.

2. Pagano L, Pulsoni a, Vignetti M, et al. Acute megakaryoblastic leukemia: experience of GIMEMA trials. Leukemia 16: 16221626, 2002.

3. Giri S, Pathak R, Prouet P, Li B, Martin MG. Acute megakaryocytic leukemia is associated with worse outcomes than other types of acute myeloid leukemia. Blood 124: 3833-3835, 2014.

4. Oki Y, Kantarjian HM, Zhou X, et al. Adult acute megakaryocytic leukemia: an analysis of 37 patients treated at M.D. Anderson Cancer Center. Blood 107: 880-885, 2006.

5. Kantarjian H, Ravandi F, O'Brien $S$, et al. Intensive chemotherapy does not benefit most older patients (age 70 years or older) with acute myeloid leukemia. Blood 116: 4422-4429, 2010.

6. Fenaux P, Mufti GJ, Hellstrom-Lindberg E, et al. Efficacy of azacitidine compared with that of conventional care regimens in the treatment of higher-risk myelodysplastic syndromes: a randomised, open-label, phase III study. Lancet Oncol 10: 223-232, 2009.

7. Quintás-Cardama A, Ravandi F, Liu-Dumlao T, et al. Epigenetic therapy is associated with similar survival compared with intensive chemotherapy in older patients with newly diagnosed acute myeloid leukemia. Blood 120: 4840-4845, 2012.

8. van der Helm LH, Veeger NJ, Kooy Mv, et al. Azacitidine results in comparable outcome in newly diagnosed AML patients with more or less than $30 \%$ bone marrow blasts. Leuk Res 37: 877882, 2013.

9. Thépot S, Itzykson R, Seegers V, et al. Azacitidine in untreated acute myeloid leukemia: a report on 149 patients. Am J Hematol 89: 410-416, 2014.

10. Dombret H, Seymour JF, Butrym A, et al. International phase 3 study of azacitidine vs conventional care regimens in older patients with newly diagnosed AML with $>30 \%$ blasts. Blood 126: 291-299, 2015.

11. Gumy-Pause F, Wacker P, Sappino AP. ATM gene and lymphoid malignancies. Leukemia 18: 238-242, 2004.

12. Austen B, Skowronska A, Baker C, et al. Mutation status of the residual ATM allele is an important determinant of the cellular response to chemotherapy and survival in patients with chronic lymphocytic leukemia containing an 11q deletion. J Clin Oncol 25: 5448-5457, 2007.

13. Haidar MA, Kantarjian H, Manshouri T, et al. ATM gene deletion in patients with adult acute lymphoblastic leukemia. Cancer 88: 1057-1062, 2000.

14. Dastugue N, Lafage-Pochitaloff M, Pagès MP, et al. Cytogenetic profile of childhood and adult megakaryoblastic leukemia (M7): a study of the Groupe Franais de Cytogénétique Hématologique (GFCH). Blood 100: 618-626, 2002.

15. Greenberg PL, Tuechler H, Schanz J, et al. Revised international prognostic scoring system for myelodysplastic syndromes. Blood 120: 2454-2465, 2012.

16. Koenecke C, Göhring G, de Wreede LC, et al. Impact of the revised International Prognostic Scoring System cytogenetics and monosomal karyotype on outcome after allogeneic stem cell transplantation for myelodysplastic syndromes and secondary acute myeloid leukemia evolving from myelodysplastic syndromes. Haematologica 100: 400-408, 2015.

17. Wang SA, Abruzzo LV, Hasserjian RP, et al. Myelodysplastic syndromes with deletions of chromosome 11q lack cryptic $M L L$ rearrangement and exhibit characteristic clinicopathologic features. Leuk Res 35: 351-357, 2011.

18. Klampfl T, Milosevic JD, Puda A, et al. Complex patterns of chromosome 11 aberrations in myeloid malignancies target CBL, MLL, DDB1 and LMO2. PLoS One 8: e77819, 2013.

19. Viniou N, Terpos E, Rombos J, et al. Acute myeloid leukemia in a patient with ataxia-telangiectasia: a case report and review of the literature. Leukemia 15: 1668-1670, 2001.

20. Lin $\mathrm{CH}$, Lin WC, Wang $\mathrm{CH}$, et al. Child with ataxia telangiectasia developing acute myeloid leukemia. J Clin Oncol 28: e213-e214, 2010.

21. Brioli A, Parisi S, Iacobucci I, et al. Patient with ataxia telangiectasia who developed acute myeloid leukemia. Leuk Lymphoma 52: 1818-1820, 2011.

22. Lu Y, Huang Q. A unique lethal blastoid transformation of B-cell chronic lymphocytic leukemia carrying ATM/11q deletion and trisomy 12. Leuk Res 33: e124-e126, 2009.

23. Greipp PT, Smoley SA, Viswanatha DS, et al. Patients with chronic lymphocytic leukaemia and clonal deletion of both $17 \mathrm{p}$ 13.1 and 11q22.3 have a very poor prognosis. Br J Haematol 163: 326-333, 2013.

24. Gardiner A, Parker H, Glide S, et al. A new minimal deleted region at $11 \mathrm{q} 22.3$ reveals the importance of interpretation of diminished FISH signals and the choice of probe for ATM deletion screening in chronic lymphocytic leukemia. Leuk Res 36: 307310,2012

25. Khanna KK, Jackson SP. DNA double-strand breaks: signaling, repair and the cancer connection. Nat Genet 27: 247-254, 2001.

26. Imanishi S, Umezu T, Ohtsuki K, Kobayashi C, Ohyashiki K, Ohyashiki JH. Constitutive activation of the ATM/BRCA1 pathway prevents DNA damage-induced apoptosis in 5-azacytidineresistant cell lines. Biochem Pharmacol 89: 361-369, 2014.

27. Ramos F, Thépot S, Pleyer L, et al. Azacitidine frontline therapy for unfit acute myeloid leukemia patients: clinical use and outcome prediction. Luek Res 39: 296-306, 2015.

(C) 2016 The Japanese Society of Internal Medicine http://www.naika.or.jp/imonline/index.html 